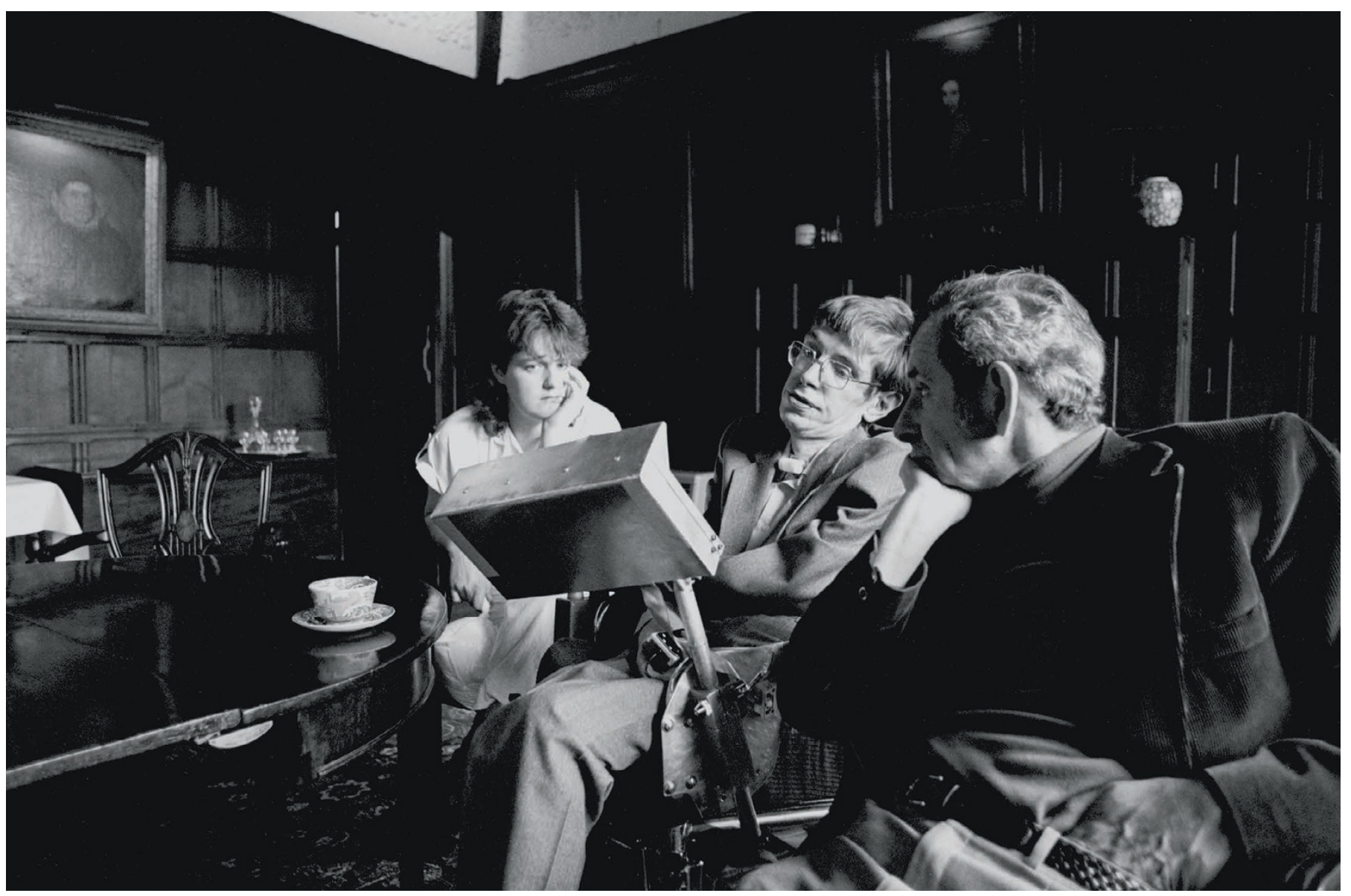

Stephen Hawking and colleagues at the University of Cambridge in the 1980 s, before the publication of A Brief History of Time.

PUBLISHING

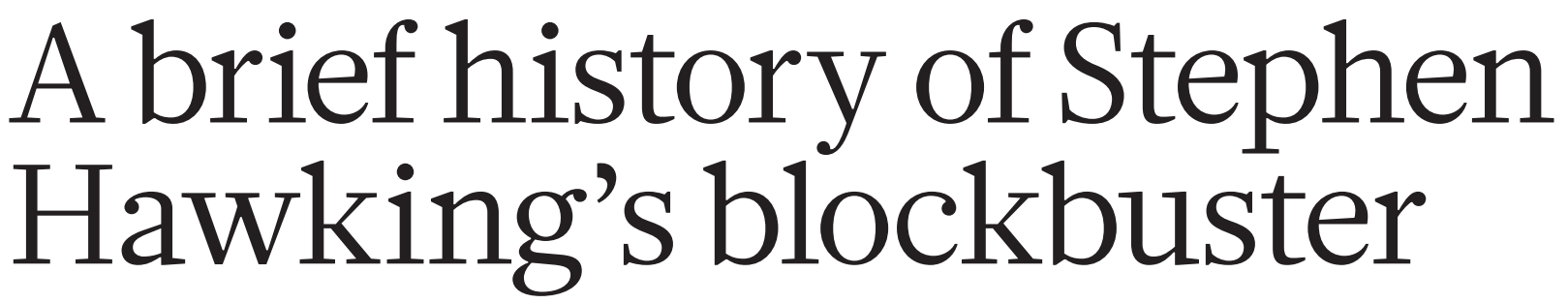

Elizabeth Leane surveys the extraordinary influence of the physicist's first foray into popular-science publishing.

$\mathrm{T}$ owards the end of The Theory of Everything, the 2014 film about Stephen Hawking, scenes depict the reception of his popular science classic, $A$ Brief History of Time (1988). The camera zooms in on a large display of the book in a shop window; fans crowd the physicistauthor, hoping that he will autograph their copies. Future events are outlined in the film's closing titles: the first relates not to the ongoing impact of Hawking's scientific achievements or the challenges of living with motor neuron disease, but to the sales of the book, which by 2013 stood at more than 10 million.

Sales of popular-science books very rarely reach the million mark, and are usually much lower. So this is an astounding achievement for a science book aimed at the non-scientist, and especially for one that grapples with the some of the biggest questions in physics - the Big Bang, black holes, a 'theory of everything' and the nature of time. As Hawking entertainingly related in a 2013 essay in The Wall Street Journal, he rewrote A Brief History repeatedly at the behest of his editor, Peter Guzzardi at Bantam, to make it more understandable to a lay readership. He later regretted, however, not further clarifying tough concepts such as imaginary time.

As a publishing phenomenon, A Brief
History of Time is not, as is sometimes claimed, unprecedented. There were nineteenth-century science blockbusters such as mathematician Mary Somerville's 1834 On the Connexion of the Physical Sciences (see R. Holmes Nature 514, 432433 ; 2014). And in 1930, physicist James Jeans' The Mysterious Universe achieved a comparable reception to Hawking's book (in Britain, at least); the jacket of the 1937 Pelican edition promotes it as "the famous book which upset tradition by making Science a bestseller". Nor was Hawking writing in a vacuum. The 1970s and 1980s had seen a series of big-selling popularphysics books, from Fritjof Capra's 1975 
The Tao of Physics to Steven Weinberg's 1977 The First Three Minutes, Carl Sagan's Cosmos (1980) and James Gleick's Chaos (1987). The sales of A Brief History, however, put even these best-sellers in the shade.

Hawking's book changed perceptions of the market for popular science. Keen to repeat his success, US and UK publishers in the late 1980s and early 1990s invested heavily in the genre. They quickly promoted existing titles (Penguin, for example, reissued physicist Paul Davies's backlist with rebranded covers); signed on new ones, in some cases with inflated advances; and opened popular-science lists. Bookshops placed slick displays of popularscience titles in prominent positions, and publishing-industry magazines started talking about a 1990s 'popular-science book boom. Titles by newcomers to the field such as Steven Pinker and Jared Diamond sold well, as did new releases by established popularizers such as Stephen Jay Gould and Richard Dawkins. With exact figures difficult to access, it is unclear to what degree a boom empirically existed, but the genre's profile had undeniably risen.

\section{HARD ACT TO FOLLOW}

Attempts to capitalize on the 'Hawking phenomenon' went hand in hand with speculations about the factors that led to it. Hawking was highly regarded in the physics community, and had been a minor celebrity to the general public even before his watershed book, appearing (for example) in Nigel Calder's UK television series The Key to the Universe in the late 1970s. The disparity between the physical limitations of his disability and the cosmic scale of his ideas was part of his charisma. Few other popular-physics books of the period feature, as A Brief History does, a photograph of the author on the front.

As Hawking himself noted, the book's title was also important. Guzzardi convinced him to revise his original suggestion, 'From the Big Bang to Black Holes: A Short History of Time'. 'Brief' is much better than 'short' in this context because it suggests duration - a human-scale interval that produces an ironic, and striking, juxtaposition with the abstract concept of time. Framing time as a historical phenomenon has a similar effect. And in the text itself, rhetorical gestures towards fundamental philosophical and theological issues - to uncover a theory of everything, writes Hawking, would be to "know the mind of God" - beckon a broad readership. But although these ingredients were readily identified, Hawking's

\section{SNATURE.COM} For more on science in culture see: nature.com/ booksandarts

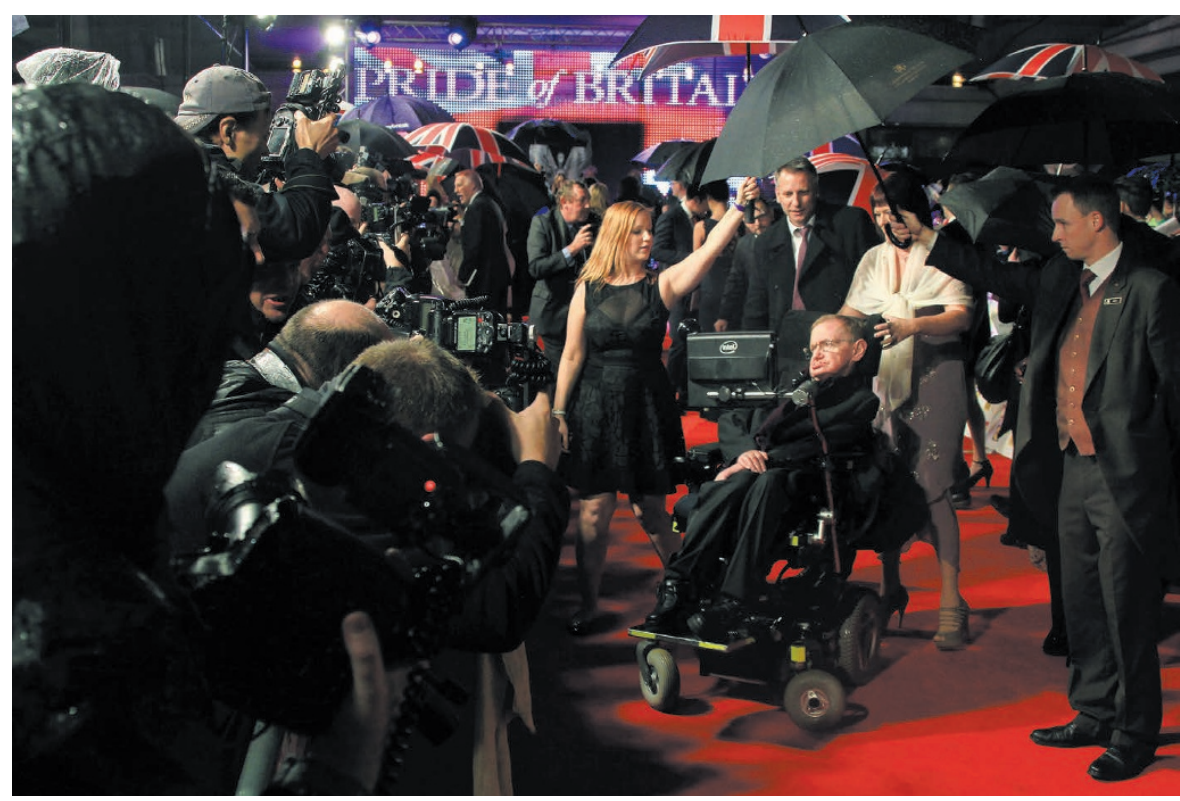

Hawking's book contributed to his becoming one of the world's biggest science celebrities.

recipe could not be repeated, even in his own subsequent books.

Most commentators agreed on what was not relevant to sales: A Brief History's readability. Despite the rewrites, it has a certain notoriety as the book everyone bought and no one read. There is now even a light-hearted 'Hawking index' designed to measure just how much a particular best-seller is read: A Brief History scores low (as, more surprisingly, does the 2011 Fifty Shades of Grey). Evidently, the act of buying Hawking's book (or E. L. James's, for that matter) says something about the consumer's identity; reading it is secondary.

\section{LONE GENIUS}

Other runaway popular-science best-sellers did follow. Dava Sobel's 1995 Longitude, Simon Singh's 1997 Fermat's Last Theorem and Brian Greene's The Elegant Universe (1999) all appeared towards the end of the last millennium. It is difficult, however, to identify any commonality with Hawking's success, except perhaps an emphasis on the figure of the scientist, broadly speaking. Sobel's text concentrates on a "lone genius", the clockmaker John Harrison. Singh's features one mathematician in its title and cover image, and focuses closely on another in its narrative. The Elegant Universe's popular success was spurred on by its adaptation as a US television series featuring the charismatic Greene.

A few years into the new millennium, however, commentators were agreeing that the 'boom' had subsided. The large sales of Bill Bryson's 2003 A Short History of Nearly Everything, with its Hawkingesque title, seemed as much a product of the travel writer's established following as of its topic.
Standout 'serious' non-fiction best-sellers now tended to come from the social sciences, with Malcolm Gladwell's 2000 The Tipping Point and the 2005 Freakonomics by Steven Levitt and Stephen Dubner achieving worldwide success.

A Brief History did, however, leave another important legacy to popular science: a new sense of the cultural capital of scientific ideas. From the 1990s onwards, space in broadsheets that had been devoted to liter-

"Although the
ingredients
were readily
identified,
Hawling's
recipe could not
be repeated."

ary texts opened up to scientific ones. Popularizers began to speak regularly at cultural festivals. Science book prizes were inaugurated, funded and maintained. The lives of scientists and mathematicians became suitable subjects for mainstream films, such as Ron Howard's 2001 A Beautiful Mind (about mathematician John Nash); The Imitation Game, the 2014 biopic of computing pioneer Alan Turing; and, indeed, The Theory of Everything.

A Brief History of Time was not solely responsible for these developments. But it gave science's presence in the cultural sphere an enormous boost: the landscape for popular-science writing was changed irrevocably by Hawking's unpredictable triumph.

Elizabeth Leane holds degrees in physics and English literature. She is currently an associate professor of English at the University of Tasmania in Hobart, Australia. Her books include Reading Popular Physics and South Pole: Nature and Culture.

e-mail:elizabeth.leane@utas.edu.au 